\title{
Advances in intravesical therapy for the treatment of non-muscle invasive bladder cancer (Review)
}

\author{
MICHAEL D. WEINTRAUB, QINGDI QUENTIN LI and PIYUSH K. AGARWAL \\ Urologic Oncology Branch, Center for Cancer Research, National Cancer Institute, \\ National Institutes of Health, Bethesda, MD 20892, USA
}

Received April 2, 2014; Accepted May 21, 2014

DOI: $10.3892 / \mathrm{mco} .2014 .314$

\begin{abstract}
The knowledge of tumor biology and the biomechanical properties of the urothelium have led to significant advances in the development of intravesical therapy for the treatment of non-muscle invasive bladder cancer (NMIBC). Targeted therapy improves the efficacy and decreases the side effects of antineoplastic agents. Nanoparticles that target antitumor agents to the urothelial cells have allowed for improved delivery of these agents to tumor cells. Gene therapy is another strategy that has allowed for a targeted induction of an antitumor response. Finally, engineering of the bacillus Calmette-Guérin (BCG) vaccine aimed to minimize the potential side effects associated with this treatment. These novel approaches hold promise for decreasing the rate of progression and recurrence of NMIBC.
\end{abstract}

\section{Contents}

1. Introduction

2. Advances in drug delivery vectors

3. Advances in gene therapy

4. BCG-based therapies

5. Conclusion

\section{Introduction}

The estimated incidence of bladder cancer in the United States (US) was 72,570 in 2013, with 15,210 expected deaths from bladder cancer. Bladder cancer is associated with the highest cost among all types of cancer, due to the need for lifelong

Correspondence to: Dr Qingdi Quentin Li or Dr Piyush Agarwal, Urologic Oncology Branch, Center for Cancer Research, National Cancer Institute, National Institutes of Health, 10 Center Drive, MSC 1210, Building 10, Bethesda, MD 20892, USA

E-mail: liquenti@mail.nih.gov

E-mail: agarwalpk2@mail.nih.gov

Key words: bladder cancer, urothelial carcinoma, intravesical therapy, targeted therapy, bacillus Calmette-Guérin routine monitoring and treatment (1). At diagnosis, $70 \%$ of bladder cancers are non-muscle invasive (NMIBC). Of these, $70 \%$ are stage Ta (papillary tumors confined to the urothelium), $20 \%$ are $\mathrm{T} 1$ (invading the lamina propria) and $10 \%$ are carcinoma in situ (CIS). NMIBC is currently treated using a combination of transurethral resection (TURBT) and intravesical therapy, depending on the stage and grade of the tumor. The main concern regarding NMIBC is the risk of progression to muscle invasive disease. Although the recurrence rate is high in both low- and high-grade disease, the risk of progression to muscle invasion depends primarily on tumor grade (2). In patients with CIS, which is high-grade by definition, the rate of progression is $>50 \%$ (3). However, low-grade Ta tumors rarely progress, although they recur at a significantly higher rate. While a number of intravesical agents are available for the treatment of NMIBC, there is a need for development of novel therapies that minimize the side effects and maximize the benefits of treatment, notably reducing disease recurrence and preventing progression.

The standard-of-care therapy for NMIBC is TURBT immediately followed by a single intravesical instillation of a chemotherapeutic agent, usually mitomycin $\mathrm{C}$, to decrease the risk of recurrence (4). Repeat TURBT is indicated for pT1 and high-grade Ta tumors, as the rate of upstaging is high (5). Intravesical immunotherapy with bacillus Calmette-Guérin (BCG) has been reported to be the most effective treatment for CIS, reducing the rate of disease recurrence and progression; BCG has also been shown to decrease progression and recurrence rates when administered after TURBT for T1 lesions (6). BCG is administered as a 6-week induction dose followed by multiple 3-week maintenance doses. The protocols for BCG therapy vary, although the most common is the one employed in the 2000 SWOG study, in which maintenance treatment is administered at 3 months, at 6 months and subsequently every 6 months for 3 years following TURBT (7).

Although BCG therapy is currently considered the most effective treatment for NMIBC, it is often associated with side effects. Fever is the most common side effect, occuring in $75 \%$ of the patients, whereas gross hematuria occurs in $25 \%$ of the patients and other side effects, including irritative voiding symptoms, arthralgias and granulomatous prostatitis, occur at a lower frequency (8). The most feared side effect of BCG is the development of BCG sepsis, which may be life-threatening, and occurs in $>0.1 \%$ of the patients (8). While 
intravesical delivery of BCG serves to limit its systemic spread, thus decreasing the risk of sepsis, the risk is increased in cases of traumatic catheterization or active infection of the bladder; therefore, BCG administration is delayed in such cases.

Considering the health and financial burden of NMIBC, there is an unmet need for the development of more effective treatment modalities to prevent disease progression and recurrence. Intravesical therapy is an attractive approach, as it has the potential to reduce the side effects associated with systemic drug absorption, thus allowing for a broader therapeutic window. Furthermore, intravesical therapy allows for more targeted delivery of the therapeutic compound to the tumor cells located in the bladder epithelium. However, intravesical therapy has to overcome two major physiological hurdles. The first one is a limited dwell time of drug in the bladder, due to washout during voiding; the second is the limited uptake of the drug into the urothelial cells (normal or malignant), due to the unique properties of the urothelium, as described below.

A number of targeted approaches are currently under evaluation for use in intravesical therapy, with the aim to develop an approach that will maximize delivery of the therapeutic compound directly to the tumor cells, while minimizing systemic absorption. To this end, constructs have been created in which vectors that target the urothelium are conjugated to chemotherapeutic or immunotherapeutic agents with known or hypothesized efficacy in bladder cancer. A number of these approaches are discussed below.

\section{Advances in drug delivery vectors}

The urothelium provides a water-tight barrier that prevents leakage of urine from the intravesical space (9). The urothelium is composed of three cell layers: a basal germinal cell layer, an intermediate cell layer and the superficial 'umbrella cell' layer. The 'umbrella cells' are joined by tight junctions, preventing passive diffusion of substances across the urothelial cell layer. On their apical surface, the umbrella cells express a high density of plaques composed of uroplakins. These plaques provide mechanical strength to the epithelial barrier. In addition, the luminal urothelial surface is covered by a continuous mucin layer composed of glycosaminoglycans (GAGs). These hydrophilic molecules form a stationary aqueous layer covering the luminal surface of the urothelium, serving as a barrier between the bladder lumen and the urothelial cell layer. The GAG mucin layer prevents the adhesion of intravesical substances to the umbrella cell layer, limiting absorption of intravesical foreign substances. Therefore, improved penetration of the therapeutic agents into the bladder wall requires mechanical or chemical disruption of the intravesical permeability barrier composed of the umbrella cell tight junctions and the GAG mucin layer.

One approach to increase drug bioavailability has focused on using lipid nanoparticles (LNPs) enclosing the compound of interest. In one study, a LNP was used to deliver small activating RNAs (saRNAs) to the tumor cells. saRNAs are a recently characterized class of double-stranded RNA molecules, which have been shown to induce RNA expression by mechanisms including transcriptional activation. It is known that one of the most common genetic derangements in bladder cancer is inactivation of the p53 tumor suppressor gene.
Suppression of the TP53 protein, in turn, leads to decreased expression of $\mathrm{p} 21$, a cyclin-dependent kinase inhibitor. A saRNA to p21 (dsP21-322-2'F) was formulated, which was intravesically delivered via LNP into nude mice, reportedly achieving a $40 \%$ tumor regression rate in the treated mice (10).

A number of studies have focused on docetaxel, which is widely used in cancer therapy and has shown some efficacy in metastatic bladder cancer. Docetaxel is a chemotherapeutic agent belonging to the taxane group, which acts by stabilizing microtubules and inducing cell cycle arrest. Barlow et al (11) conducted a phase I trial of intravesical docetaxel in patients with BCG-refractory NMIBC. In that study, 54 patients with BCG-refractory NMIBC received 6 weekly instillations of intravesical docetaxel. Of these patients, 18 received further maintenance therapy. A total of 59\% of the patients exhibited a complete response after this induction therapy. The 5-year disease-specific and overall survival rates were 85 and $71 \%$, respectively. In initial responders, the median time to recurrence with and without maintenance docetaxel was 39.3 and 19.0 months, respectively.

In a preclinical study, mucoadhesive nanoparticles derived from hyperbranched polyglycerols (HPG-C $8 / 10-\mathrm{MePEG}-\mathrm{NH}_{2}$ ) were used to improve the delivery of docetaxel to tumor cells, based on the increased aqueous solubility and mucoadhesive qualities of this vector (12). The authors of that study demonstrated that the docetaxel-loaded nanoparticles (DTX) significantly inhibited tumor growth in vivo compared to controls and to intravesical administration of commercially available docetaxel (Taxotere) and also demonstrated that the DTX formulation increased the permeability of the urothelial layer and increased drug uptake into the tumor tissue. Based on these results, nanoparticulated docetaxel may be an effective delivery system for the treatment of BCG-refractory NMIBC.

Nanoparticle albumin-bound agents (NAB) have been extensively investigated for improving the targeting of chemotherapeutic agents. NAB-paclitaxel has been approved by the US Food and Drug Administration as systemic therapy for metastatic breast cancer, as it demonstrated increased efficacy and a more favorable side effect profile compared to standard paclitaxel in a phase III trial (13). The intravesical administration of NAB-paclitaxel in NMIBC was evaluated in a phase I trial, the results of which were recently reported (14). A total of 18 patients with high-grade Ta, T1, or transitional CIS, who had failed at least one previous course of intravesical therapy, were recruited in that study. No grade 2,3 , or 4 toxicities were observed and 5 of the 18 patients (28\%) had no evidence of disease at 6 weeks post-treatment. A phase II trial of NAB-paclitaxel is underway. Additionally, a phase I study of NAB-rapamycin (ABI-009) was recently conducted on patients with advanced solid malignancies (ClinicalTrials. gov; no. NCT00635284), the results of which are pending.

\section{Advances in gene therapy}

Adenoviral-mediated gene transfer has been extensively investigated as targeted therapy in a variety of cancers, including bladder cancer. A number of bladder tumor cells express the coxsackie virus and adenovirus receptor, making adenoviral targeting of drugs to the urothelium an attractive gene transfer technology (15). A recombinant adenovirus (rAd) 
carrying the interferon- $\alpha 2 b$ (IFN- $\alpha$ ) gene was developed to transduce the IFN- $\alpha$ gene into urothelial cells. Recombinant IFN- $\alpha$ has been clinically shown to improve the efficacy of BCG in combination treatment (16). For this reason, IFN- $\alpha$ is an attractive candidate for gene therapy in BCG-refractory NMIBC. To increase binding to the urothelium and penetration across the urothelial cell surface, the rAd-IFN- $\alpha$ vector was associated with the excipient SCH 209702 (Syn3), which has been shown to enhance adenoviral-mediated transduction in NMIBC (17). In a phase I trial of 17 patients with NMIBC who had experienced BCG failure, no dose-limiting toxicity was encountered (18). Moreover, $43 \%$ of the patients experienced a complete response at 3 months and 2 patients remained disease-free at 29 and 32 months, respectively. A multi-institutional phase II trial, assessing the efficacy and safety of rAd-IFN- $\alpha$ with Syn3 in treating BCG-refractory of relapsed NMIBC, is currently underway (ClinicalTrials.gov; no. NCT01687244).

A second approach using adenoviral-mediated gene transfer in BCG-refractory NMIBC has been investigated. In this approach, a replication-selective serotype-5 oncolytic adenovirus, CG0070, was used to transduce the granulocyte-monocyte colony-stimulating factor (GM-CSF) gene into tumor cells. CG0070 was engineered to preferentially replicate in and destroy retinoblastoma (RB) protein-defective cells (19). Thus, it was hypothesized that the anticancer effects of this therapy may be due to the direct oncolytic effect of the vector, as well as the immunostimulatory effect of GM-CSF. In a phase I trial, 35 patients with at least one failure of BCG treatment received either single or multiple infusions of CG0070 at different doses (20). The complete response rate was $48.6 \%$ across all cohorts, with a median duration of complete response of 10.4 months. A $63.6 \%$ complete response rate was observed in the multi-dose cohort. Of note, the complete response rate among patients who were retrospectively found to have borderline or high RB phosphorylation and who received a multi-dose regimen, was $81.8 \%$. An integrated phase II/III trial, assessing the efficacy of CG0070 expressing GM-CSF in BCG-failure patients, is currently underway (ClinicalTrials. gov; no. NCT01438112).

Adenoviral-mediated gene transfer has also been used to deliver rapamycin, a mammalian target of rapamycin (mTOR) inhibitor, to a genetically-engineered mouse model of CIS progressing to muscle-invasive disease (21). This mouse model exhibited combinatorial deletion of the p53 and PTEN genes, a genotype shared with several high-grade human bladder cancers. It was demonstrated that deletion of these genes leads to activation of the mTOR signaling pathway and is correlated with progression from CIS to MIBC (22). Intravescial rapamycin delivery using an adenoviral vector expressing Cre recombinase in this mouse model of CIS decreased the progression rate, as well as tumor size. In the same study, it was demonstrated that intravesical administration of rapamycin was more efficacious when compared to intravenous delivery.

In another approach to gene therapy for urothelial carcinoma, plasmids coding for cyclooxygenase-2 (COX-2)-inducible caspase-3 and -9 were intravesically delivered in a mouse model of urothelial carcinoma (23). This model exploited the fact that bladder cells have been shown to overexpress COX-2 (24). The plasmids were complexed to a polymer, polycation poly(ethylenimine), which was shown to increase the permeability of the urothelial barrier. The results of this study demonstrated an inhibition of tumor progression in treated mice. The authors of that study hypothesized that this was due to either a pro-apoptotic or anti-angiogenic effect of the induction treatment.

\section{BCG-based therapies}

Building on the efficacy of BCG therapy in NMIBC, investigators have looked into developing treatments that elicit an immune response comparable to that of BCG, which is a live attenuated vaccine, while avoiding the systemic side effects of BCG. Mycobacterial cell wall extract (MCWE) from Mycobacterium phlei is similar in composition to BCG but does not contain live bacteria, therefore potentially avoiding systemic toxicity. MCWE was originally found to be efficient in BCG-refractory CIS and was associated with a favorable toxicity profile in a previous multicenter clinical trial (25). However, the MCWE emulsion used in that study contained a preservative named thiomersal, which is a mercury derivative of known toxicity. One method of overcoming this issue is through the use of the mycobacterial cell wall-DNA complex (MCC), in which mycobacterial DNA is complexed to cell wall fragments. It was demonstrated that MCC exerts its anticancer effects by directly inducing apoptosis and indirectly stimulating the immune system (26). A multi-institutional clinical trial evaluated MCC in BCG-naïve and -refractory patients with CIS (27). Of the patients receiving a higher treatment dose $(8 \mathrm{mg}), 46 \%$ achieved a complete response at 12 weeks post-treatment. At 18 months, $29 \%$ of these patients remained disease-free. Severe adverse events occurred in $33 \%$ of the patients; however, only one patient required treatment discontinuation. Based on these promising results, a phase III trial assessing the use of MCC in BCG-refractory CIS or recurrent high-grade NMIBC within 2 years of successful treatment with BCG, is currently underway (ClinicalTrial. gov; no. NCT00406068). Treatment-related adverse events have been reported in $86 \%$ of patients in this trial, with 2 patients requiring treatment discontinuation due to adverse effects. Interim results indicated a certain efficacy of MCC in BCG-refractory patients, although repeat BCG induction was found to be more effective (28). Therefore, this therapy may not be as promising as initially anticipated.

Targeted therapy of BCG components has also been investigated. One group of investigators used an octaarginine-modified liposome (R8-liposome) vector conjugated to non-live BCG cell wall skeleton (R8-liposome-BCG-CWS). The authors had previously demonstrated that R8-liposomes attached to tumor cells and effectively released their contents in the cell interior; upregulation of natural killer cells and stimulation of a Th1 response with administration of this compound was also reported (29). In rats treated with N-butyl-N-(4-hydroxybutyl)-nitrosamine, a known bladder carcinogen, the intravesical administration of R8-liposome-BCG-CWS resulted in decreased rate of tumor growth (30).

Other investigators have developed recombinant BCG vaccines with the goal of increasing the pro-inflammatory Th1 immune response elicited by BCG. In one such study, 
rBCG-S1PT, a recombinant BCG vaccine expressing the pertussis toxin subunit $\mathrm{S} 1$ was administered to mice following intravesical tumor implantation (31). That study demonstrated a decrease in mean tumor weight and an increase in survival of the mice treated with $\mathrm{rBCG}-\mathrm{S} 1 \mathrm{PT}$ compared to those treated with BCG. An increase in the expression of tumor necrosis factor- $\alpha$ mRNA in the rBCG-S1PT-treated mice was also observed, which is indicative of an enhanced Th1 response as a result of this treatment.

Finally, investigators at the National Cancer Institute have initiated a study in BCG-refractory patients, randomizing them to BCG alone vs. BCG with a subcutaneous vaccine. This vaccine, PANVAC, is a poxviral cancer vaccine that has demonstrated therapeutic efficacy against a variety of carcinomas. PANVAC comprises a 'priming vaccine', consisting of a replication-competent recombinant vaccinia vector, followed by 'booster vaccines' consisting of replication-incompetent recombinant fowlpox vector. These vectors contain transgenes for the tumor-associated antigens carcinoembryonic antigen and mucin-1, which are overexpressed in high-grade bladder cancer. This vaccine also has three co-stimulatory molecules that may enhance the immune response. That trial is currently being conducted as a phase I/II study (ClinicalTrials.gov; NCT no. NCT02015104).

\section{Conclusion}

NMIBC remains a challenging clinical entity, due to the lack of effective therapeutic options and the high risk of progression and recurrence despite treatment. Although BCG-based therapy has been the mainstay of treatment for CIS of the bladder for over three decades, its use is limited by potentially life-threatening side effects and by the fact that several tumors are resistant or refractory to treatment with BCG. Therefore, the development of intravesical therapies is an attractive approach, which has the potential to directly target urothelial tumor cells, while avoiding systemic side effects. However, a major limiting factor in intravesical therapy is the urothelial permeability barrier, which limits the tissue penetration of drugs. Therefore, a number of the current approaches in intravesical therapy rely on vectors that either target the active compound to the urothelial cell surface or are able to better penetrate the urothelial barrier due to their chemical structure. The agents used in these novel approaches include traditional chemotherapeutic agents, such as docetaxel, paclitaxel, or rapamycin.

The other approach is gene therapy, which targets genetic dysfunctions that have been shown to be specific to NMIBC. A number of these approaches have already demonstrated proof of principle and are currently in clinical trials. By combining novel treatment modalities with enhanced methods of drug delivery to the urothelium, effective therapeutic options may finally become available to patients with NMIBC to limit disease recurrence and progression to muscle-invasive disease.

\section{Acknowledgements}

This study was supported by the Intramural Research Program of the National Cancer Institute, the National Institutes of Health, USA.

\section{References}

1. Botteman MF, Pashos CL, Redaelli A, Laskin B and Hauser R: The health economics of bladder cancer: a comprehensive review of the published literature. Pharmacoeconomics 21: 1315-1330, 2003.

2. Millan-Rodriguez F, Chechile-Toniolo G, Salvador-Bayarri J, Palou $\mathrm{J}$ and Vicente-Rodriguez J: Multivariate analysis of the prognostic factors of primary superficial bladder cancer. J Urol 163: 73-78, 2000.

3. Donat SM: Evaluation and follow-up strategies for superficial bladder cancer. Urol Clin North Am 30: 765-776, 2003.

4. Witjes JA and Hendricksen K: Intravesical pharmacotherapy for non-muscle-invasive bladder cancer: a critical analysis of currently available drugs, treatment schedules, and long-term results. Eur Urol 53: 45-52, 2008.

5. Schwaibold HE, Sivalingam S, May F and Hartung R: The value of a second transurethral resection for T1 bladder cancer. BJU Int 97: 1199-1201, 2006.

6. Cookson MS and Sarosdy MF: Management of stage T1 superficial bladder cancer with intravesical bacillus Calmette-Guerin therapy. J Urol 148: 797-801, 1992.

7. Lamm DL, Blumenstein BA, Crissman JD, et al: Maintenance bacillus Calmette-Guerin immunotherapy for recurrent TA, T1 and carcinoma in situ transitional cell carcinoma of the bladder: a randomized Southwest Oncology Group Study. J Urol 163: 1124-1129, 2000

8. Lamm DL: Efficacy and safety of bacille Calmette-Guérin immunotherapy in superficial bladder cancer. Clin Infect Dis 31 (Suppl 3): S86-S90, 2000.

9. GuhaSarkar S and Banerjee R: Intravesical drug delivery: challenges, current status, opportunities and novel strategies. J Control Release 148: 147-159, 2010.

10. Kang MR, Yang G, Place RF, et al: Intravesical delivery of small activating RNA formulated into lipid nanoparticles inhibits orthotopic bladder tumor growth. Cancer Res 72: 5069-5079, 2012.

11. Barlow L, McKiernan JM and Benson MC: Long-term survival outcomes with intravesical docetaxel for recurrent nonmuscle invasive bladder cancer after previous bacillus Calmette-Guérin therapy. J Urol 189: 834-839, 2013.

12. Mugabe C, Matsui Y, So AI, et al: In vivo evaluation of mucoadhesive nanoparticulate docetaxel for intravesical treatment of non-muscle-invasive bladder cancer. Clin Cancer Res 17: 2788-2798, 2011.

13. Gradishar WJ, Tjulandin S, Davidson N, et al: Phase III trial of nanoparticle albumin-bound paclitaxel compared with polyethylated castor oil-based paclitaxel in women with breast cancer. J Clin Oncol 23: 7794-7803, 2005.

14. McKiernan JM, Barlow LJ, Laudano MA, Mann MJ, Petrylak DP and Benson MC: A phase I trial of intravesical nanoparticle albumin-bound paclitaxel in the treatment of bacillus Calmette-Guérin refractory nonmuscle invasive bladder cancer. J Urol 186: 448-451, 2011.

15. Buscarini M, Quek ML, Gilliam-Hegarich S, Kasahara N and Bochner B: Adenoviral receptor expression of normal bladder and transitional cell carcinoma of the bladder. Urol Int 78: 160-166, 2007.

16. O'Donnell MA, Lilli K, Leopold C, et al: Interim results from a national multicenter phase II trial of combination bacillus Calmette-Guerin plus interferon alfa-2b for superficial bladder cancer. J Urol 172: 888-893, 2004.

17. Yamashita M, Rosser CJ, Zhou JH, et al: Syn3 provides high levels of intravesical adenoviral-mediated gene transfer for gene therapy of genetically altered urothelium and superficial bladder cancer. Cancer Gene Ther 9: 687-691, 2002.

18. Dinney CP, Fisher MB, Navai N, et al: Phase I trial of intravesical recombinant adenovirus mediated interferon- $\alpha 2 b$ formulated in Syn3 for Bacillus Calmette-Guérin failures in nonmuscle invasive bladder cancer. J Urol 190: 850-856, 2013.

19. Ramesh N, Ge Y, Ennist DL, Zhu M, Mina M, Ganesh S, Reddy PS and Yu DC: CG0070, a conditionally replicating granulocyte macrophage colony-stimulating factor - armed oncolytic adenovirus for the treatment of bladder cancer. Clin Cancer Res 12: 305-313, 2006.

20. Burke JM, Lamm DL, Meng MV, et al: A first in human phase 1 study of CG0070, a GM-CSF expressing oncolytic adenovirus, for the treatment of nonmuscle invasive bladder cancer. J Urol 188: 2391-2397, 2012. 
21. Seager CM, Puzio-Kuter AM, Patel T, et al: Intravesical delivery of rapamycin suppresses tumorigenesis in a mouse model of progressive bladder cancer. Cancer Prev Res (Phila) 2: 1008-1014, 2009.

22. Puzio-Kuter AM, Castillo-Martin M, Kinkade CW, et al: Inactivation of p53 and Pten promotes invasive bladder cancer. Genes Dev 23: 675-680, 2009.

23. Zhang X and Godbey WT: Preclinical evaluation of a gene therapy treatment for transitional cell carcinoma. Cancer Gene Ther 18: 34-41, 2011.

24. Mohammed SI, Knapp DW, Bostwick DG, et al: Expression of cyclooxygenase-2 (COX-2) in human invasive transitional cell carcinoma (TCC) of the urinary bladder. Cancer Res 59: 5647-5650, 1999.

25. Morales A, Chin JL and Ramsey EW: Mycobacterial cell wall extract for treatment of carcinoma in situ of the bladder. J Urol 166: 1633-1638, 2001.

26. Filion MC and Phillips NC: Therapeutic potential of mycobacterial cell wall-DNA complexes. Expert Opin Investig Drugs 10: $2157-2165,2001$
27. Morales A, Phadke K and Steinhoff G: Intravesical mycobacterial cell wall-DNA complex in the treatment of carcinoma in situ of the bladder after standard intravesical therapy has failed. J Urol 181: 1040-1045, 2009.

28. Ahn JJ and McKiernan JM: New agents for bacillus Calmette-Guérin-refractory bladder cancer. Urol Clin North Am 40: 219-232, 2013.

29. Miyazaki J, Kawai K, Kojima T, et al: The liposome-incorporating cell wall skeleton of Mycobacterium bovis bacillus Calmette-Guérin can directly enhance the susceptibility of cancer cells to lymphokine-activated killer cells through up-regulation of natural-killer group 2, member D ligands. BJU Int 108: 1520-1526, 2011.

30. Miyazaki J, Nishiyama H, Yano I, et al: The therapeutic effects of R8-liposome-BCG-CWS on BBN-induced rat urinary bladder carcinoma. Anticancer Res 31: 2065-2071, 2011.

31. Andrade PM, Chade DC, Borra RC, et al: The therapeutic potential of recombinant BCG expressing the antigen S1PT in the intravesical treatment of bladder cancer. Urol Oncol 28: 520-525, 2010. 